\title{
SCIENTIFIC REPORTS

\section{Correlative Study of Enhanced Excitonic Emission in ZnO Coated with Al Nanoparticles using Electron and Laser Excitation}

\author{
Saskia Fiedler ${ }^{1,2^{*}}$, Laurent O. Lee Cheong Lem ${ }^{1,3}$, Cuong Ton-That ${ }^{1}$, Markus Schleuning ${ }^{4}$, \\ Axel Hoffmann ${ }^{4}$ \& Matthew R. Phillips ${ }^{1 *}$
}

Recently, metal nanoparticle surface coatings have been found to significantly enhance the ultra-violet luminescence intensity from $\mathrm{ZnO}$, providing a viable means to mitigate optical losses and improve LED performance. Although there is general agreement that resonantly excited Localized Surface Plasmons (LSPs) in metal nanoparticles can directly couple to excitons in the semiconductor increasing their spontaneous emission rate, the exact mechanisms involved in this phenomenon are currently not fully understood. In this work, LSP-exciton coupling in bulk and nanostructured ZnO coated with a $2 \mathrm{~nm}$ Al nanoparticle layer is investigated using correlative photoluminescence and depth-resolved cathodoluminescence and time-resolved photoluminescence spectroscopy. Temperature-resolved cathodoluminescence and photoluminescence measurements from $10 \mathrm{~K}$ to $250 \mathrm{~K}$ show free exciton (FX) emission enhancement factors up to $12 x$ at $80 \mathrm{~K}$, and reveal that the FX couple more efficiently to the LSPs compared to the localized donor-bound excitons. A strong polarization dependence between the LSPs and FX is observed where FX transitions are more strongly enhanced when polarized in the same direction as the electric field of the incident excitation, which is different for laser and electron beam sources. This result indicates that selective enhancement of the excitonic emission peaks in the ZnO coated with Al nanoparticles can be achieved by choosing the appropriate $\mathrm{ZnO}$ substrate orientation.

Despite LEDs exhibiting very high internal quantum efficiencies, only a small fraction of the total light generated is emitted due to significant optical reflection losses arising from large refractive index differences at the air/semiconductor interface. Improved light extraction from LEDs device structures will increase their overall performance, in particular their energy efficiency. Localized surface plasmon (LSP) - exciton coupling has been widely reported as a means to significantly enhance the luminescence of $\mathrm{ZnO}$ and other semiconductors by using a metal nanoparticle (NP) surface coating ${ }^{1-8}$. LSPs greatly enhance the local electric field at the surface of the metal of NPs via the collective oscillation of free electrons in the presence of an external electromagnetic field. Significantly, when the plasma resonance energy of the metal NPs matches the excitonic energy of the semiconductor, LSPs can efficiently couple to excitons in semiconductors. This LSP resonance energy is dependent on the type of metal employed, the dimension and the shape of the NPs as well as the permittivity of the surrounding dielectric. Silver

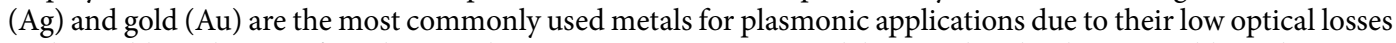
in the visible and near infrared spectral regions. However, Au is widely considered to be unsuitable in the UV as it has high losses for energies above $2.3 \mathrm{eV}$ due to its interband transitions. Although Ag seems to be eminently suitable for UV plasmonic applications as its interband transitions occur at higher optical energies, this is not the case as it is much less stable in air than Au. Specifically, Ag readily forms oxide and sulphur layers when exposed to air making the fabrication of stable plasmonic Ag NPs difficult ${ }^{9,10}$. Conversely, Aluminum (Al) affords more robust NPs due to strong self-limiting oxidation effects even in very small particles ${ }^{11}$. Moreover, Al NPs have the advantage of a widely size tunable plasmon resonance energy from the deep UV to the visible spectral range as

${ }^{1}$ School of Mathematical and Physical Sciences, University of Technology Sydney, 15 Broadway, Ultimo, NSW, 2007, Australia. ${ }^{2}$ Centre for Nano Optics, University of Southern Denmark, Campusvej 55, 5230, Odense M, Denmark. ${ }^{3}$ Australian National Fabrication Facility, Australian National University, Canberra, ACT 2601, Australia. ${ }^{4}$ Technische Universität Berlin, Fakultät II, Institut für Festkörperphysik, Sekretariat EW 5-4, Hardenbergstr. 36, 10623, Berlin, Germany.*email: safi@mci.sdu.dk; matthew.phillips@uts.edu.au 


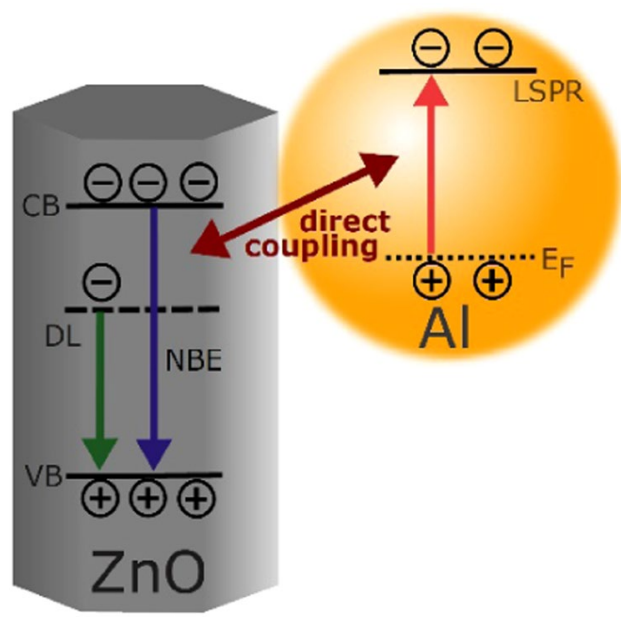

Figure 1. Illustration of the LSP-exciton coupling mechanism.

well as being cheaper and more abundant than both $\mathrm{Au}$ and $\mathrm{Ag}^{12-15}$. These attributes of Al NPs are clearly highly desirable for plasmonic applications in the blue-UV spectral range, in particular, the enhancement of the excitonic luminescence output in $\mathrm{ZnO}$ based opto-electronic devices.

$\mathrm{ZnO}$ is a wide-bandgap semiconductor $(\mathrm{Eg}=3.376 \mathrm{eV}$ at $4 \mathrm{~K})$ with a large exciton binding energy of $60 \mathrm{meV}^{16-18}$. A typical $\mathrm{ZnO}$ luminescence spectrum consists of a sharp UV emission at around $3.37 \mathrm{eV}$ and a broad visible defect-related emission positioned between 2.3 and $1.7 \mathrm{eV}^{18}$. The $\mathrm{UV}$ emission at $4 \mathrm{~K}$ originates from excitonic recombination involving three free excitons $(\mathrm{FX})\left(\mathrm{E}_{\mathrm{A}}=3.3781 \mathrm{eV} ; \mathrm{E}_{\mathrm{B}}=3.3856 \mathrm{eV}\right.$; $\mathrm{E}_{\mathrm{C}}=3.4262 \mathrm{eV}$ ) due to split valance band of $\mathrm{ZnO}$ as well as a number of donor-bound excitons (DBX), arising from impurities, such as $\mathrm{H}, \mathrm{Al}, \mathrm{Ga}$ and $\mathrm{In}^{19-21}$. The polarization of the FX-A and FX-B in wurtzite $\mathrm{ZnO}$ is different to FX-C: The FX-A and FX-B are perpendicularly polarized to the $c$-axis, while FX-C is parallel polarized $^{22-24}$. As the temperature is increased from $80 \mathrm{~K}$ to $300 \mathrm{~K}$, the DBXs thermally ionize and quench while the FX and its LO phonon replicas with a $72 \mathrm{meV}$ spacing red shift, become wider and overlap to produce a broad near band edge emission centered at $3.30 \mathrm{eV}$.

The $\mathrm{ZnO}$ UV emission is greatly enhanced by the addition of a thin Al NP surface coating as the excitons in $\mathrm{ZnO}$ can directly couple to the LSPs in the Al NP. In this process, the presence of an additional, non-radiative, faster relaxation LSP channel results in an increased spontaneous emission rate and consequently, an enhanced UV excitonic luminescence intensity. This LSP-exciton coupling mechanism in an Al-coated ZnO nanorod (NR) is illustrated in Fig. 1. Although the LSP-exciton coupling between $\mathrm{ZnO}$ and $\mathrm{Al}$ interpretation for the increased emission seems plausible and is widely accepted, there is an inexplicably large range of enhancement factors reported in the literature, extending over orders of magnitude ${ }^{1,3,25-30}$. To address this issue, an extensive investigation of the coupling mechanism in $\mathrm{AlNP}$ coated $\mathrm{ZnO}$ has been conducted.

In this work, two types of Al-coated $\mathrm{ZnO}$ samples, $a$-plane $\mathrm{ZnO}$ single crystals and a vapor-solid (VS)-grown $\mathrm{ZnO}$ NR ensemble, were systematically studied using photoluminescence (PL) spectroscopy and depth-resolved cathodoluminescence (CL) spectroscopy at temperatures ranging from $10 \mathrm{~K}$ to $250 \mathrm{~K}$. An up to 12 times enhanced UV PL emission of Al-coated $\mathrm{ZnO}$ was typically observed compared with their uncoated counterparts. In comparison, an 8 times enhancement was measured with a CL excitation of $3 \mathrm{kV}$. The Al LSPs were found to couple more strongly to the mobile FX in $\mathrm{ZnO}$ compared with localized DBX with the greatest enhancement observed with exciton excitation close to the surface.

Additionally, the LSP-exciton coupling enhancement strength of the FX-A/FX-B and FX-C emissions was found to be a dependent on the crystallographic orientation of the $\mathrm{ZnO}$ sample relative to the Al NP LSP dipole direction, which is mediated by the electric field alignment of the excitation, which is different for laser and electron beam sources. Differences in the measured excitonic UV emission enhancement between PL and CL excitation mechanisms are attributed to variation in the FX-LSP coupling strength caused by different: (i) FX generation depths relative to the NP-ZnO surface and (ii) orientation of the LSP dipole relative to the surface plane.

\section{Results and Discussion}

Depth-resolved CL spectra of the uncoated and Al-coated $\mathrm{ZnO}$ at a temperature of $10 \mathrm{~K}$ and $80 \mathrm{~K}$ are shown in Fig. 2. At both temperatures, an enhanced UV emission from the Al-coated side of the sample is observed compared to the adjacent uncoated $\mathrm{ZnO}$ control region. The UV enhancement factor is obtained by dividing the integrated UV emission from $3.00 \mathrm{eV}$ to $3.45 \mathrm{eV}$ from the Al-coated $\mathrm{ZnO}$ by that of the uncoated side of the sample. At a temperature of $10 \mathrm{~K}$, the UV emission enhancement factor at $3 \mathrm{kV}$ and $5 \mathrm{kV}$ is found to be 1.8 , which reduced to 1.5 at an acceleration voltage of $10 \mathrm{kV}$. A higher $\mathrm{UV}$ enhancement close to the $\mathrm{ZnO}-\mathrm{Al}$ interface is found at $80 \mathrm{~K}$ with integrated UV enhancement factors of 8.3 at $3 \mathrm{kV}, 3.3$ at $5 \mathrm{kV}$ and 2.2 at $10 \mathrm{kV}$. The Al NP surface coating induced changes in the intensity of the defect luminescence in the visible spectral region in Fig. 2 arise from surface depletion layer effects, which are unrelated to the observed UV enhancement. This increased near-surface enhancement effect at $80 \mathrm{~K}$ is illustrated in Fig. 3, which shows the CL enhancement factor as a function of emission energy at $3 \mathrm{kV}, 5 \mathrm{kV}$ and $10 \mathrm{kV}$. Here, the dashed vertical lines show the FX-related peaks in 

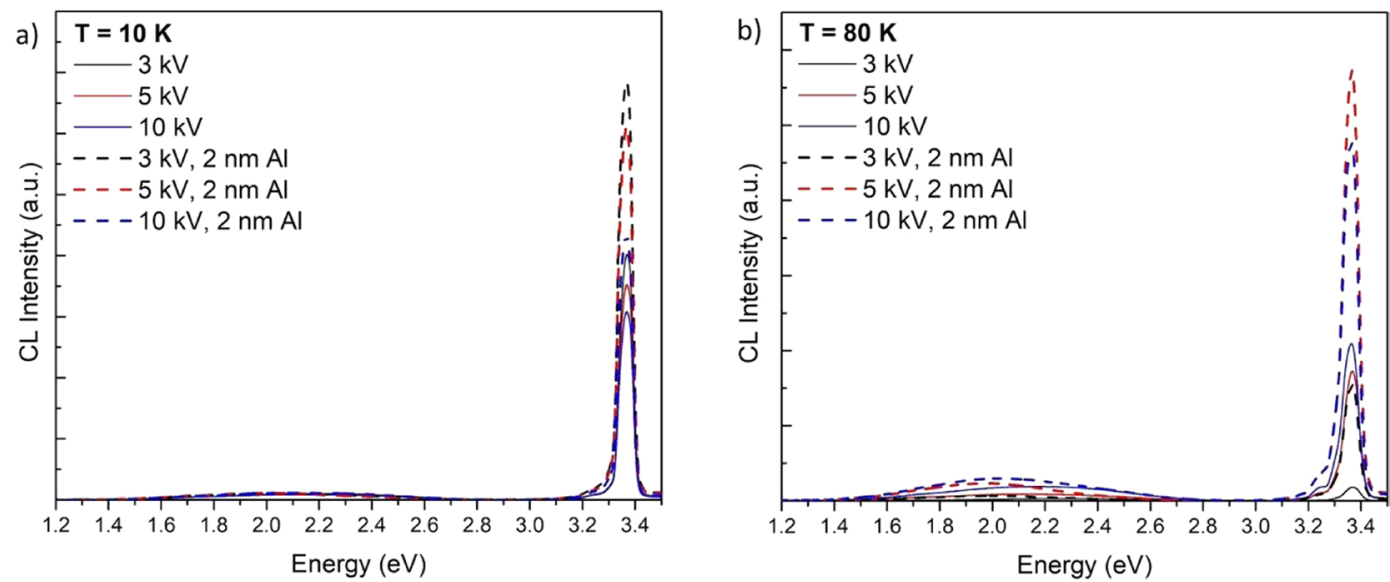

Figure 2. Depth-resolved CL UV-Vis full spectra of uncoated (solid lines) and Al-coated ZnO (dashed lines) at a temperature of (a) $10 \mathrm{~K}$ and (b) $80 \mathrm{~K} . \boldsymbol{P}=17.5 \mu \mathrm{W}$ with varying accelerating voltage of $3 \mathrm{kV}, 5 \mathrm{kV}$ and $10 \mathrm{kV}$, corresponding to approximate CL generation depths of $40 \mathrm{~nm}, 100 \mathrm{~nm}$ and $350 \mathrm{~nm}$.

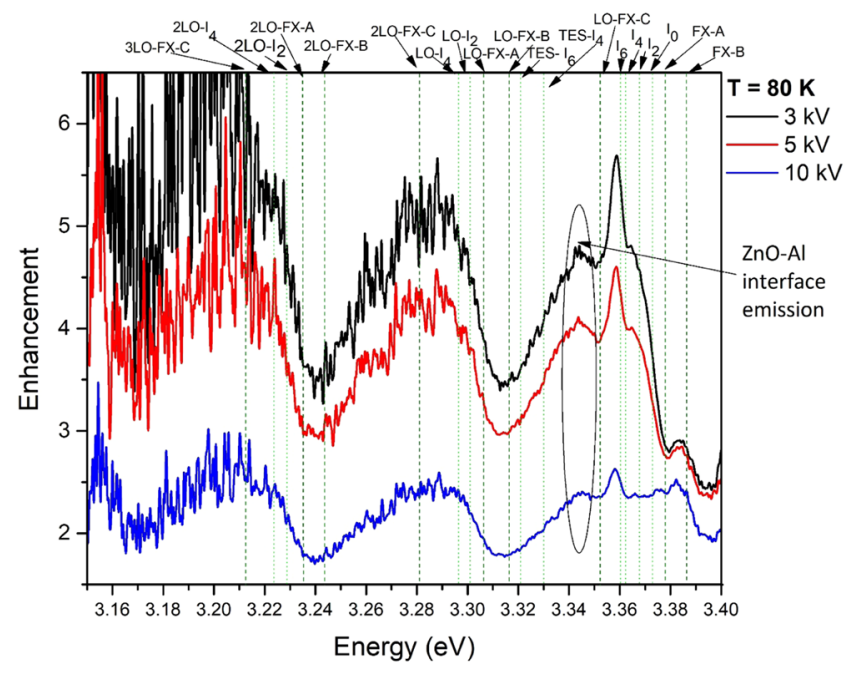

Figure 3. CL enhancement factor as a function of energy at acceleration voltages of $3 \mathrm{kV}$ (black), $5 \mathrm{kV}$ (red) and $10 \mathrm{kV}$ (blue), resulting in CL generation depths of approximately $40 \mathrm{~nm}, 100 \mathrm{~nm}$, and $350 \mathrm{~nm}$, respectively. Dotted green lines illustrate the DBX-related transitions in $\mathrm{ZnO}$, while the dashed olive lines represent the FXrelated emissions. $P=17.5 \mu \mathrm{W}, T=80 \mathrm{~K}$.

$\mathrm{ZnO}$, while the dotted lines indicate the DBX-related emissions ${ }^{20,21,31}$. Fig. 3 shows that the first three LO phonon replicas of the FX-C emission at $3.353 \mathrm{eV}, 3.280 \mathrm{eV}$ and $3.203 \mathrm{eV}$ are enhanced by the presence of the $\mathrm{Al}$ film. The plot also reveals a strong enhancement at $3.358 \mathrm{eV}$ which corresponds to the donor bound exciton, I6 line ${ }^{31}$. This emission peak has been unequivocally assigned to $\mathrm{Al}$ donor impurities in $\mathrm{ZnO}$, suggesting that some $\mathrm{Al}$ may have in-diffused into the near surface region of the crystal. The arrow label at $3.343 \mathrm{eV}$ in Fig. 3 indicates an emission which cannot be attributed to any of the reported luminescence peaks in $\mathrm{ZnO}$ and $\mathrm{Al}_{2} \mathrm{O}_{3}$. As the enhancement level of this peak is observed to increase with decreasing $\mathrm{kV}$, where carriers are injected closer to the surface, it is most likely due to an emission arising from interface related defects residing at the $\mathrm{ZnO}$ and $\mathrm{Al}$ boundary. Additionally, these results collectively confirm that the LSPs in Al couple more efficiently to the FX than to the DBX. Furthermore, the CL enhancement is temperature-dependent with the UV enhancement being greater at $80 \mathrm{~K}$ than at $10 \mathrm{~K}$.

The temperature dependence of the $\mathrm{Al}$ induced UV enhancement of the PL from $10 \mathrm{~K}$ to $250 \mathrm{~K}$ is displayed in Fig. 4. Shown at the bottom of the plot are the measured PL spectra of the uncoated and the Al-coated samples at $10 \mathrm{~K}$ used to determine the energy dependent PL enhancement in the top frame. The dashed vertical lines specify the DBX-related emissions, while the dottedlines indicate the FX peaks. The enhanced emission peak at $3.343 \mathrm{eV}$ indicated by the arrow label in Fig. 4 is consistent with the peak at the same energy observed in the CL measurements (Fig. 3) originating from radiative defects located at the $\mathrm{ZnO}-\mathrm{Al}$ interface. The enhancement is found to maximize at three energy positions that are in agreement with the positions of the FX-C-LO1, FX-C-LO2 and FX-C-LO3 excitonic emissions. Moreover, these three maxima red shift in energy as the temperature increases 


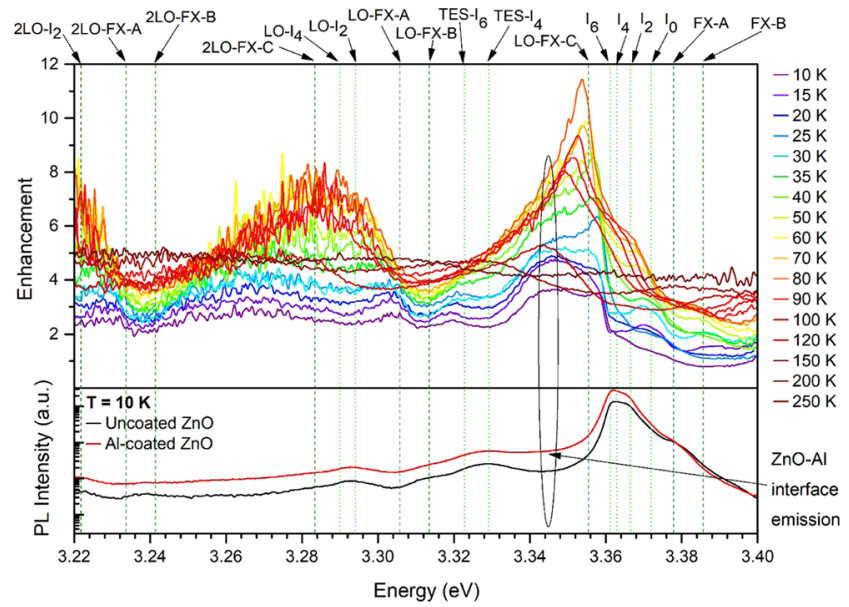

Figure 4. Bottom: PL spectra of the uncoated (black) and Al-coated (red) $\mathrm{ZnO}$ at $10 \mathrm{~K}$. Top: Temperatureresolved PL enhancement factor as a function of energy. Dottedgreen lines illustrate the DBX-related transitions in $\mathrm{ZnO}$, while the dashed olive lines represent the FX-related emissions. $P=2.1 \mathrm{~mW}$.

from $10 \mathrm{~K}-250 \mathrm{~K}$ which is consistent with the widening of band gap due to increasing electron-phonon scattering effect. The temperature-resolved data reveal that the maximum enhancement factor of $\sim 12$ was achieved at $80 \mathrm{~K}$, following the FX-C emission intensity dependence on temperature. As the temperature rises from $10 \mathrm{~K}$ to $80 \mathrm{~K}$, the competitive DBX recombination channels thermalize and quench, increasing the FX-C intensity, which then falls approaching $250 \mathrm{~K}$ as higher order exciton $\mathrm{LO}$ phonon replicas become dominant. This observation supports the assignment of the $\mathrm{Al}$ thin film induced emission enhancement to the formation of an additional relaxation channel due to LSP coupling to the FX-C.

Correlative temperature-resolved CL at $5 \mathrm{kV}$ and PL spectroscopy measurements at $10 \mathrm{~K}$ and $80 \mathrm{~K}$ are shown in Fig. 5(a,b), respectively. At 10 K, the UV enhancement factor in CL and PL is similarly low at 2.5 . While the low energy side of the spectrum of the enhancement factor appears very similar for both types of excitation, the higher energy side above $3.334 \mathrm{eV}$ shows a more pronounced UV enhancement in CL than in PL. This can be attributed to a higher contribution of the FX-A and FX-B LSP-exciton coupling when excited by the electron beam rather than by the laser. With both CL and PL excitation, greater Al LSP coupling occurs with FX compared with $\mathrm{DBX}$ in $\mathrm{ZnO}$. This is because the FX have a larger spatial extent providing a larger wave function overlap than with the DBX, which are spatially localized at the donor. A similar argument is also used to explain why FX phonon coupling is generally much stronger the DBX-phonon coupling ${ }^{32,33}$. Greater FX over DBX coupling can also be due to the FX having a high mobility enabling FX diffusion towards the $\mathrm{ZnO}$ and $\mathrm{Al}$ surface coating interface, where the LSP-FX separation is small, and coupling is strongly enhanced.

Due to the higher FX emission intensity at $80 \mathrm{~K}$, where the DBX is fully thermally dissociated, a greater coupling strength in PL and CL is observed, as shown in Fig. 5b. Here, the PL enhancement has its maximum of approximately 12 , while the maximum CL enhancement is 4 . The 3 -fold difference in CL and PL enhancement can be explained by the difference in type of excitation: electron beam or laser light. While the excitation profile of the laser is highest at the surface following the Beer-Lambert law profile with depth where approximately $8 \%$ of the total optical absorption occurs in the first $5 \mathrm{~nm}$ of the sample. In contrast, Monte Carlo CASINO modelling shows that the maximum excitation in CL is deeper in the bulk of the sample at around $1 / 3$ of the electron range. In $\mathrm{CL}$ at $5 \mathrm{kV}$, only about $2 \%$ of the energy is lost within the first $5 \mathrm{~nm}$ of the sample indicating that the generation of the FX close to the Al NP surface coating is considerably greater when excited by the laser compared with the electron beam. Therefore, the probability of LSPs in Al coupling to FX in $\mathrm{ZnO}$ is higher for PL than with CL.

Furthermore, the relative enhancement factors of FX-A, FX-B, and FX-C in Al-coated ZnO are different for laser and electron beam excitation. It is well-known that the polarization of the $\mathrm{FX}$ in $\mathrm{ZnO}$ are different for the FX-A and FX-B, which are perpendicularly polarized to the $c$-axis of $\mathrm{ZnO}$, while the FX-C is parallel polarized $^{22-24}$. This results in different LSP-exciton coupling strengths for the two different types of excitation. The polarization of the laser excitation is always perpendicular to the k-vector of the incident light; consequently, in our PL setup geometry the laser electric vector is always parallel to the sample surface. Accordingly, the surface parallel polarized LSPs in $\mathrm{Al}$, excited by the laser can couple more efficiently to the equally polarized FX-C while the perpendicular polarized FX-A and -B cannot couple as strongly. Therefore, a higher enhancement of FX-C than that of FX-A and -B can be achieved with laser light excitation. Conversely, for electron excitation with the beam normal to the surface, the incident electron and its image charge within the NP is dipole-like, producing an electric field perpendicular to the surface efficiently which excites Al NP LSPs in the same direction ${ }^{34-36}$. Consequently, LSPs in the Al NPs are excited parallel to the surface under laser excitation and perpendicular to the surface with the electron beam. The LSP dipole orientation difference between PL and CL excitation results in higher enhancement of the FXs that are polarized in the same plane as the incident electric field of the excitation source. 

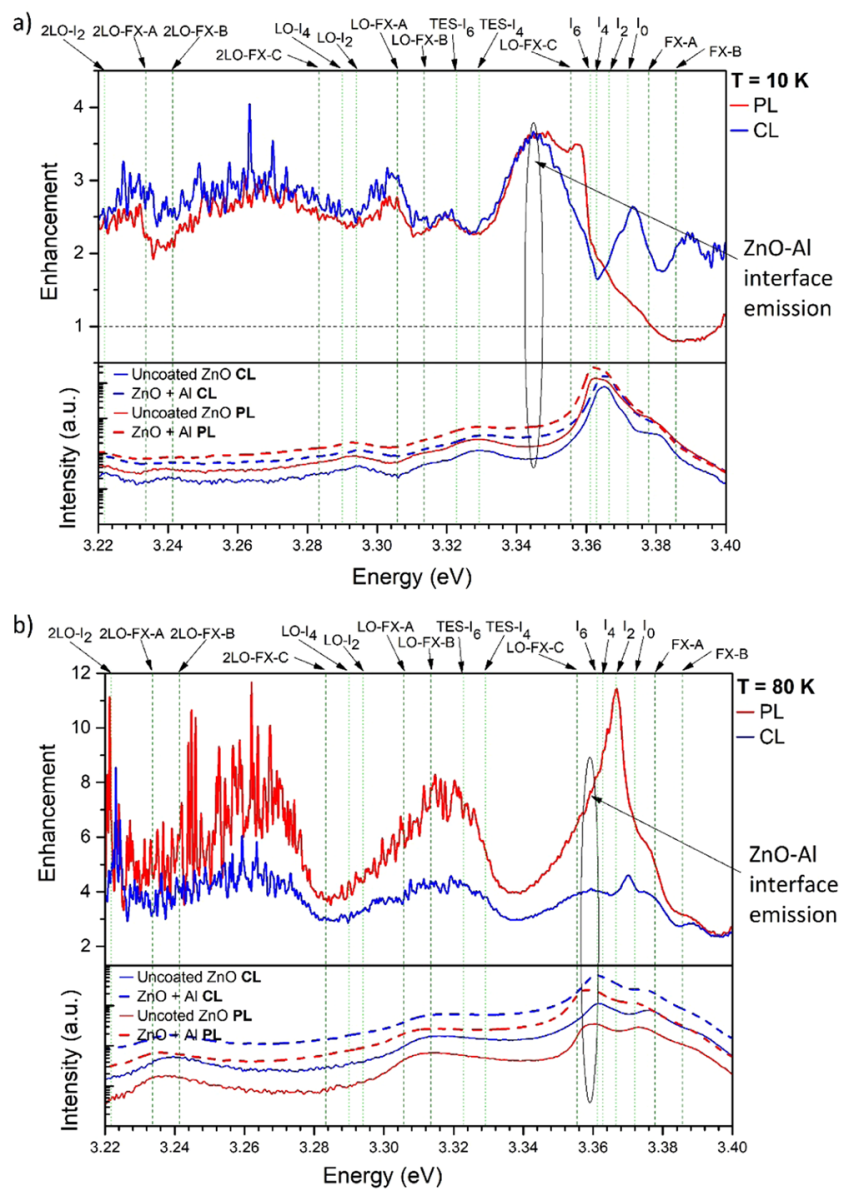

Figure 5. (a) Bottom: PL (blue) and CL (red) spectra at $5 \mathrm{kV}$ of the uncoated (solid lines) and Al-coated (dashed lines) $\mathrm{ZnO}$ at $10 \mathrm{~K}$. Top: Corresponding PL and CL enhancement factor as a function of energy. Dotted green lines illustrate the DBX-related transitions in $\mathrm{ZnO}$, while the dashed olive lines represent the FX-related emissions. (b) PL and $5 \mathrm{kV}$ CL spectra and enhancement factors at a temperature of $80 \mathrm{~K}$.

A direct comparison of the CL and PL enhancement factors as a function of energy at $10 \mathrm{~K}$ and $80 \mathrm{~K}$ is displayed in Fig. 5a,b, respectively. At $10 \mathrm{~K}$, the PL enhancement factor of the FX-C is approximately 3.5 while the FX-A and FX-B are close to 1 . At $80 \mathrm{~K}$, the PL shows an even stronger polarization effect with the FX-C being enhanced 12 times and the FX-A and FX-B showing a much lower enhancement of approximately 3 and 5, respectively. In contrast, the CL enhancement factors are found to be generally lower. Here, the Al-LSPs excited by the electron beam are polarized normal to the sample surface which is parallel to the polarization of the FX-A and FX-B but perpendicular to that of the FX-C. Thus, the combined CL enhancement of FX-A and FX-B is expected to be higher than that of FX-C. It is noteworthy that the LSP-coupling to FX-A and FX-B has a similar probability as these two relaxation channels are competing. This results in a similar polarization effect of the LSP-exciton coupling in CL as well as in PL, however, the overall enhancement is more pronounced in PL due to the near-surface excitation of the laser.

To further investigate this polarization-dependent enhancement, PL and CL measurements were taken on $c$-axis-oriented $\mathrm{ZnO}$ nanorods. Figure 6a shows the $\mathrm{SE}$ image of well-aligned $\mathrm{ZnO}$ nanorods grown by the vapor-solid (VS) method on a Si substrate. As shown in the XRD profile in Fig. SI 1, the nanowires with an average diameter of approximately $100 \mathrm{~nm}$ are grown predominantly along the (002) axis, perpendicular to the substrate. In this case, the FX-A and FX-B are parallel polarized to the sample surface while the FX-C is perpendicular polarized, resulting in the opposite polarization geometry to the $a$-plane $\mathrm{ZnO}$ single crystal discussed above. The LSP-exciton coupling of the $\mathrm{ZnO} N R$ coated with a $2 \mathrm{~nm}$ thin $\mathrm{Al}$ film (cf. above) were studied at a temperature of $80 \mathrm{~K}$ to maximize the number of FX by dissociating DBX and thereby producing a higher enhancement factor. The near-surface excitation by laser light was chosen as it was found that electron beam excitation introduced charging effects in the ZnO NR sample. Furthermore, possible excitation of LSPs in Al NPs located at the side walls of the $\mathrm{ZnO}$ NRs by a sufficiently energetic electron beam can result in a 90-degree tilted polarization state of the Al-LSPs, and therefore, lead to an overall mixed polarization of LSP-exciton coupling.

Figure $6 \mathrm{~b}$ shows the PL spectra of the uncoated and the Al-coated $\mathrm{ZnO}$ NRs at a temperature of $80 \mathrm{~K}$, as well as the resulting enhancement factor as a function of energy. The FX-A and FX-B related emissions are highly enhanced as expected since the FX-A and FX-B are polarized in the same plane as the sample parallel to the electric vector of the incident laser light. The FX-C enhancement is less pronounced as its polarization is normal to 
a)
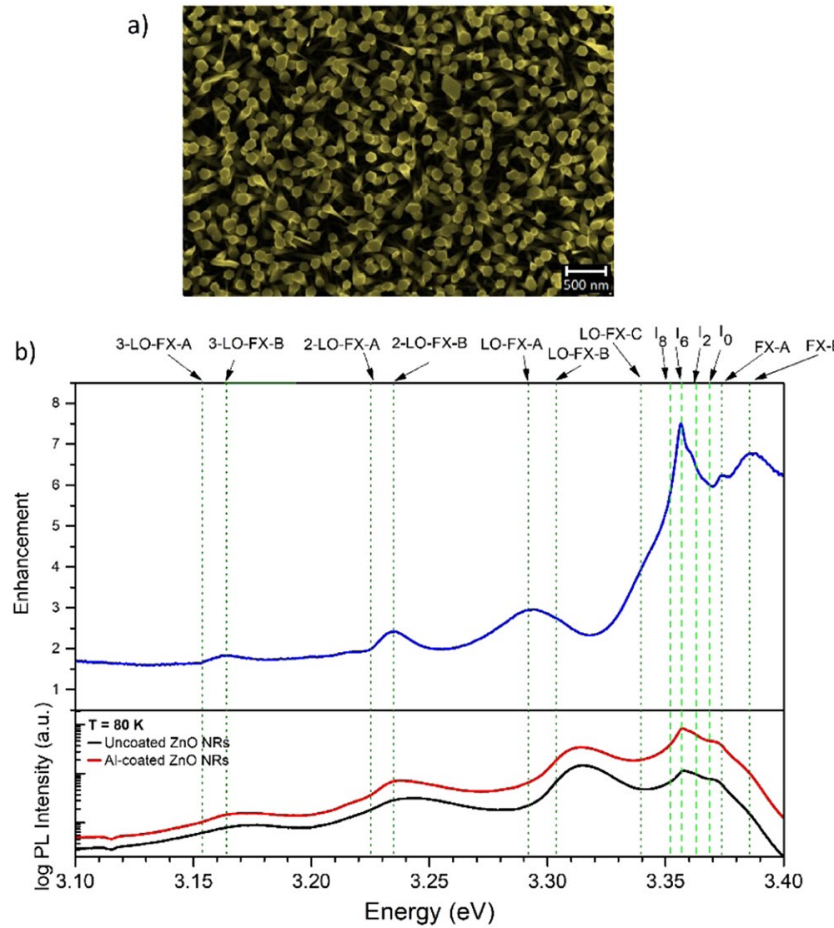

Figure 6. (a) SE image of the uncoated VS-grown $\mathrm{ZnO}$ nanorods with an average diameter of $100 \mathrm{~nm}$. (b) Bottom: PL spectra of the uncoated (black) and Al-coated (red) VS-grown $\mathrm{ZnO}$ nanorods at $80 \mathrm{~K}$. Top: Corresponding PL enhancement factor as a function of energy. Dashed green lines illustrate the DBX-related transitions in $\mathrm{ZnO}$, while the dotted olive lines represent the FX-related emissions.
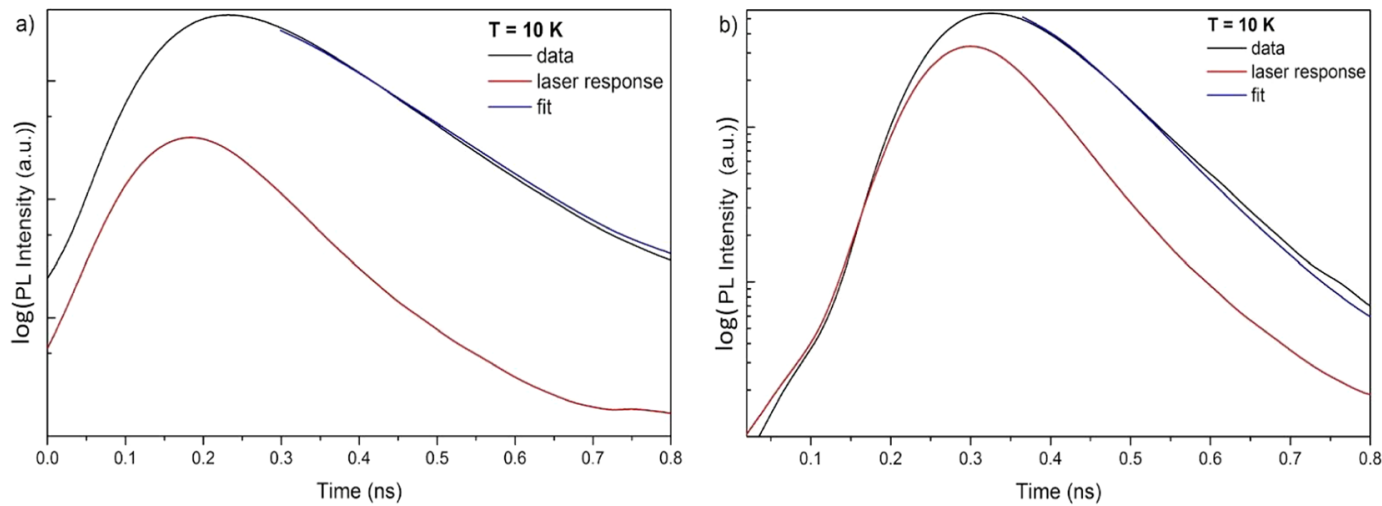

Figure 7. (a) Time-resolved PL of the uncoated $\mathrm{ZnO}$ with a lifetime of $\tau_{Z n O}=(161 \pm 4) \mathrm{ps}$ and (b) of the Alcoated $\mathrm{ZnO}$, showing a reduced lifetime of $\tau_{\mathrm{ZnO}+\mathrm{Al}} \leq 53 \mathrm{ps}$.

the $c$-plane. These polarization studies confirm that the PL coupling strength of the Al NP LSPs to the three FX transitions in $\mathrm{ZnO}$ and their emission enhancement is determined by the crystallographic orientation sample, which fixes the polarization direction of the three FX with respect to the incident laser polarization. It is also noteworthy, that the greatest PL enhancement in the VS ZnO NRs is found at the Al DBX I6 line, suggesting that Al may have diffused into the sample.

To confirm LSP-exciton coupling, TR-PL was performed on the planar a-plane $\mathrm{ZnO}$ single crystals. It is widely accepted that LSP-exciton coupling increases the spontaneous emission rate of the metal nanoparticle-coated $\mathrm{ZnO}$ through the creation of an additional, faster relaxation channel via the LSPs, leading to a reduction of the carrier lifetime in these samples ${ }^{1,37,38}$.

Figure 7 shows the time-resolved PL results for both the uncoated and Al-coated $a$-plane $\mathrm{ZnO}$ crystal collected at $10 \mathrm{~K}$. Deconvolution of the laser response with the signal was performed to obtain the fit of the relaxation curve to determine the lifetime of the emission. The uncoated side of the sample, illustrated in Fig. 7a, exhibits an excitonic lifetime of $\tau_{Z n O}=(161 \pm 4) \mathrm{ps}$, while Fig. $7 \mathrm{~b}$ displays the TR-PL results of the Al-coated $\mathrm{ZnO}$ with a lifetime of $\tau_{Z n O+A l} \leq 53 \mathrm{ps}$. The faster lifetime from the Al-coated sample is consistent with an increase in the 
spontaneous emission rate through the creation of an additional, faster relaxation channel via the LSP-exciton coupling, leading to a reduction of the carrier lifetime. The lifetime of the Al-coated $\mathrm{ZnO}$ is very close to the laser response, which does not allow the decay curve to be confidently fitted. However, a maximum lifetime of 53 ps can be used to calculate the Purcell enhancement factor, $\mathrm{F}_{\mathrm{p}}$, which quantifies the coupling strength between the LSPs in the $\mathrm{Al}$ nanoparticles and excitons in $\mathrm{ZnO}$.

$$
\mathrm{F}_{\mathrm{P}}=\tau_{\mathrm{ZnO}} / \tau_{\mathrm{ZnO}+\mathrm{Al}} \geq 3.0
$$

Due to the limited temporal resolution of the TR-PL constrained by the laser response, a lower limit of the Purcell enhancement factor was determined to be $F_{P} \geq 3.0$. A direct comparison of this $F_{P}$ value to the maximum $\mathrm{PL}$ enhancement of 12 times at a temperature of $80 \mathrm{~K}$ is not trivial due to various reasons. First, the collection of the TR-PL data was only possible at a temperature of $10 \mathrm{~K}$ where the PL spectrum of $\mathrm{ZnO}$ is dominated by the DBX which couple less effectively to the LSPs in Al than the FX. The corresponding experimentally derived PL enhancement factor is, therefore, as low as 4 (cf. Fig. 4), indicating that the calculated $\mathrm{F}_{\mathrm{p}}$ is on the same order. Second, the limited temporal resolution of the TR-PL setup only allows the determination of a lower limit of the Purcell enhancement factor not allowing for a quantitative comparison with the experiment. However, the more than three times reduced lifetime in Al-coated $\mathrm{ZnO}$ single crystals clearly confirms an increased spontaneous emission rate via LSP-exciton coupling.

\section{Conclusion}

In conclusion, LSP-exciton coupling in two types of Al-coated $\mathrm{ZnO}$ samples - $a$-plane $\mathrm{ZnO}$ single crystals and VS-grown ZnO nanorods - was studied using CL and PL spectroscopy. The results reveal that the observed enhancement is strongly dependent on the type of excitation, electron or photon, and the sample temperature. The greatest UV enhancement of approximately 12 times was observed at a temperature of $80 \mathrm{~K}$ using laser excitation. Due to the higher mobility and the larger spatial extent of the FX in $\mathrm{ZnO}$ compared with the DBX, a stronger coupling of the LSPs in the $\mathrm{Al}$ to the $\mathrm{FX}$ in $\mathrm{ZnO}$ was found. At elevated temperatures, the LSP-exciton coupling is more effective, where the spectrum is dominated by FX emissions as the DBX are mostly thermally dissociated. $\mathrm{CL}$ spectroscopy confirmed that the maximum enhancement was observed at the surface close to the $\mathrm{ZnO}-\mathrm{Al}$ interface with a higher enhancement factor at $80 \mathrm{~K}$ than at low temperatures.

Furthermore, it was demonstrated that the $\mathrm{ZnO}$ crystal orientation with respect to the electric vector orientation of the incident laser light determines which of the three FX transitions are coupled more strongly to the LSPs. The strongest PL enhancement of the FX emission was observed when the FX polarization in the same plane as the electric vector of the incident laser excitation, which is different for laser and electron beam sources.

We believe this previously unreported finding is significant as it opens the door for the selective metal NP surface coating induced enhancement of the energy and polarization of the excitonic emission in $\mathrm{ZnO}$ and similar wide band gap semiconductors, such as GaN.

\section{Methods}

Fabrication of $\mathrm{ZnO}$ samples. In this work, hydrothermally grown, $0.5 \mathrm{~mm}$ thick, $10 \times 10 \mathrm{~mm} a$-plane $\mathrm{ZnO}$ single crystal polished plates were obtained from MTI Corp (USA).

Hexagonal $\mathrm{ZnO} \mathrm{NR}$ ensembles with a mean diameter of $\sim 100 \mathrm{~nm}$ and a length of $\sim 1500 \mathrm{~nm}$ were grown on $a$-plane sapphire substrates using a vapor solid technique, which is described in detail elsewhere ${ }^{39}$.

Both the single crystal and $\mathrm{ZnO} N R$ samples were sputter-coated at room temperature with a $2 \mathrm{~nm}$ thin $\mathrm{Al}$ film. The resulting surface coating consists of metallic Al NPs embedded in an $\mathrm{Al}_{2} \mathrm{O}_{3}$ matrix as shown by ellipsometry and a reduced UV transmission (see Fig. SI 2). Prior to deposition, the single crystal samples were subjected to a standard cleaning procedure consisting of a 20-minute sonication in acetone, then isopropyl alcohol and lastly deionized water. Only half of each sample was coated with Al to leave the other, uncoated side as a control reference to facilitate a direct before and after deposition comparison of the luminescence intensity.

Optical characterization. CL and PL spectroscopy were performed in a FEI Quanta 200 SEM equipped with a Gatan CF302 continuous flow liquid helium cold stage, allowing for temperature-dependent measurements from $10 \mathrm{~K}$ to room temperature. Light injection optics allow sequential CL and PL measurements on spatially equivalent regions of the samples without breaking the SEM vacuum. PL was excited with the $325 \mathrm{~nm}$ line of a Melles Griot He-Cd laser with a power of $2.1 \mathrm{~mW}$. CL and PL emitted from the sample were collected by a parabolic mirror and analyzed using two optical spectrometers. An Ocean Optics QE Pro spectrometer with a $\sim 1 \mathrm{~nm}$ spectral resolution facilitated concurrent measurement of the ZnO UV near band edge and deep level emission in the visible spectral range. While high resolution $<0.1 \mathrm{~nm}$ spectra were measured with an Oriel MS257 $1 / 4 \mathrm{~m}$ imaging spectrograph equipped with a Hamamatsu S7011-1007 CCD. All spectra were corrected for the total response of the system. SEM acceleration voltages of $3 \mathrm{kV}, 5 \mathrm{kV}$, and $10 \mathrm{kV}$ were used for depth-resolved CL analysis and the SEM beam power was kept constant at $17.5 \mu \mathrm{W}$ by varying the electron beam current ${ }^{40}$. The Monte Carlo CASINO simulation package was used to determine the in-depth spatial distribution of the injected electron hole pairs ${ }^{40}$. For SEM acceleration voltages of $3 \mathrm{kV}, 5 \mathrm{kV}$ and $10 \mathrm{kV}$, the simulations reveal CL generation depths of approximately $40 \mathrm{~nm}, 100 \mathrm{~nm}$ and $350 \mathrm{~nm}$, respectively, corresponding to $70 \%$ of the total electron energy loss. At an acceleration voltage of $5 \mathrm{kV}$, the CL excitation depth is very similar to the excitation range of the $325 \mathrm{~nm}$ UV laser in $\mathrm{ZnO}$ of $\sim 100 \mathrm{~nm}$. However, the electron hole pair injection density with CL excitation is approximately three orders of magnitude larger than that of the laser at a power of $2.1 \mathrm{~mW}$.

Time-resolved PL was performed at $10 \mathrm{~K}$ and the luminescence decay was recorded by a standard photon counting technique. In these measurements, the excitation wavelength of the pulsed fiber $(\lambda=1031 \mathrm{~nm})$ laser was internally halved and subsequently frequency-doubled to $\lambda=258 \mathrm{~nm}$ with a pulse duration of $5.5 \mathrm{fs}$ at repetition rate of $76 \mathrm{MHz}$. 
Received: 14 October 2019; Accepted: 13 January 2020;

Published online: 13 February 2020

\section{References}

1. Lu, J. et al. Direct Resonant Coupling of Al Surface Plasmon for Ultraviolet Photoluminescence Enhancement of ZnO Microrods. ACS Appl. Mater. Interfaces 6, 18301-18305 (2014).

2. Lu, J. et al. Plasmon-enhanced Electrically Light-emitting from ZnO Nanorod Arrays/p-GaN Heterostructure Devices. Sci. Rep. 6, 25645 (2016)

3. $\mathrm{Lu}$, J. et al. Improved UV photoresponse of $\mathrm{ZnO}$ nanorod arrays by resonant coupling with surface plasmons of $\mathrm{Al}$ nanoparticles. Nanoscale 7, 3396-3403 (2015).

4. Zhang, Y. et al. Effects of localized surface plasmons on the photoluminescence properties of Au-coated ZnO films. Opt. Express 17, 8735-8740 (2009).

5. Gogurla, N. et al. Multifunctional Au-ZnO Plasmonic Nanostructures for Enhanced UV Photodetector and Room Temperature NO Sensing Devices. Sci. Rep. 4, 6483 (2014).

6. Cheng, C. W. et al. Surface plasmon enhanced band edge luminescence of $\mathrm{ZnO}$ nanorods by capping Au nanoparticles. Appl. Phys. Lett. 96, 071107 (2010).

7. Yin, J. et al. Effect of the surface-plasmon-exciton coupling and charge transfer process on the photoluminescence of metal-semiconductor nanostructures. Nanoscale 5, 4436 (2013).

8. Bertoni, G. et al. Nanoscale mapping of plasmon and exciton in ZnO tetrapods coupled with Au nanoparticles. Sci. Rep. 6, 19168 (2016).

9. Hillman, C. et al. Silver and Sulfur: Case Studies, Physics, and Possible Solutions SMTA Inter (2007).

10. Erol, M. et al. SERS Not To Be Taken for Granted in the Presence of Oxygen. J. Am. Chem. Soc. 131, 7480-7481 (2009).

11. Cabrera, N. et al. Theory of the Oxidation of Metals. Rep. Prog. Phys. 12, 163 (1949).

12. Knight, M. W. et al. Aluminum Plasmonic Nanoantennas. Nano Lett. 12, 6000-6004 (2012).

13. Gérard, D. et al. Aluminium plasmonics. J. Phys. Appl. Phys. 48, 184001 (2015).

14. Langhammer, C. et al. Localized Surface Plasmon Resonances in Aluminum Nanodisks. Nano Lett. 8, 1461-1471 (2008).

15. Taguchi, A. et al. Tailoring plasmon resonances in the deep-ultraviolet by size-tunable fabrication of aluminum nanostructures. Appl. Phys. Lett. 101, 081110 (2012).

16. Thomas, D. G. The exciton spectrum of zinc oxide. J. Phys. Chem. Solids 15, 86-96 (1960).

17. Miller, P. Zinc Oxide: A spectroscopic investigation of bulk crystals and thin films. (2008).

18. Özgür, Ü. et al. A comprehensive review of ZnO materials and devices. J. Appl. Phys. 98, 041301 (2005).

19. Wagner, M. R. et al. Bound excitons in ZnO: Structural defect complexes versus shallow impurity centers. Phys. Rev. B 84 (2011).

20. Wagner, M. R. Fundamental properties of excitons and phonons in $\mathrm{ZnO}$ : A spectroscopic study of the dynamics, polarity, and effects of external fields. (2010).

21. Liang, W. Y. et al. Transmission Spectra of ZnO Single Crystals. Phys. Rev. Lett. 20, 59-62 (1968).

22. Toropov, A. A. et al. Temperature-dependent polarized luminescence of exciton polaritons in a ZnO film. Phys. Status Solidi A 202, 392-395 (2005)

23. Mang, A. et al. Crystal-field splitting, spin-orbit coupling, and exciton binding energies in $\mathrm{ZnO}$ under hydrostatic pressure. Solid State Commun. 94, 251-254 (1995).

24. Calleja, J. M. et al. Resonant Raman scattering in ZnO. Phys. Rev. B 16, 3753-3761 (1977).

25. Ding, X. et al. Influence of Al capping on the photoluminescence of ZnO. Opt. Mater. Express 7, 1898 (2017).

26. Wu, K. et al. Enhanced near band edge emission of $\mathrm{ZnO}$ via surface plasmon resonance of aluminum nanoparticles. J. Appl. Phys. 110,023510 (2011).

27. Liu, M. et al. Tuning the influence of metal nanoparticles on $\mathrm{ZnO}$ photoluminescence by atomic-layer-deposited dielectric spacer. Nanophotonics 2 (2013).

28. Lai, C. W. et al. Surface-plasmon-mediated emission from metal-capped ZnO thin films. Appl. Phys. Lett. 86, 251105 (2005).

29. Lu, J. et al. Plasmon-mediated exciton-phonon cupling in a ZnO microtower cavity. J Mater Chem C 4, 7718-7723 (2016).

30. Dixit, T. et al. Role of Surface Plasmon Decay Mediated Hot Carriers toward the Photoluminescence Tuning of Metal Coated ZnO Nanorods. J Phys Chem C 121, 3540-3548 (2017).

31. Meyer, B. K. et al. Bound exciton and donor-acceptor pair recombinations in ZnO. Phys. Status Solidi B 241, 231-260 (2004).

32. Giles, N. C. et al. Effects of phonon coupling and free carriers on band-edge emission at room temperature in $\mathrm{n}$-type $\mathrm{ZnO}$ crystals. Appl. Phys. Lett. 89, 251906 (2006).

33. Wang, L. et al. Temperature dependence of the free-exciton transition energy in zinc oxide by photoluminescence excitation spectroscopy. J. Appl. Phys. 94, 973-978 (2003).

34. Coenen, T. et al. Angle-resolved cathodoluminescence spectroscopy. Appl. Phys. Lett. 99, 143103 (2011).

35. Vesseur, E. J. R. et al. Plasmonic excitation and manipulation with an electron beam. MRS Bull. 37, 752-760 (2012).

36. Coenen, T. et al. Directional Emission from Plasmonic Yagi-Uda Antennas Probed by Angle-Resolved Cathodoluminescence Spectroscopy. Nano Lett. 11, 3779-3784 (2011).

37. Zang, Y. et al. Band edge emission enhancement by quadrupole surface plasmon-exciton coupling using direct-contact $\mathrm{Ag} / \mathrm{ZnO}$ nanospheres. Nanoscale 5, 574-580 (2013).

38. Ren, Q. J. et al. Evidence for coupling between exciton emissions and surface plasmon in Ni-coated ZnO nanowires. Nanotechnology 23, 425201 (2012).

39. Ton-That, C. et al. Luminescent properties of ZnO nanowires and as-grown ensembles. Nanotechnology 19, 415606 (2008).

40. Drouin, D. et al. CASINO V2.42-A Fast and Easy-to-use Modeling Tool for Scanning Electron Microscopy and Microanalysis Users. Scanning 29, 92-101 (2007).

\section{Acknowledgements}

The authors would like to thank Katie McBean, Geoff McCredie and Dr. Angus Gentle for the technical support at UTS, and Dr. Stefan Kalinowski for his assistance at TU Berlin. Furthermore, we would like to acknowledge financial support from the Australian Research Council (DP150103317).

\section{Author contributions}

S.F. planned and designed the experiments under supervision of M.P. und C.T.; S.F. and L.L. performed the experiments at UTS and analyzed the results, while M.S. and A.H. were responsible for the time-resolved PL at TUB. S.F. wrote the main manuscript with great support from L.L, M.P. and C.T. All authors have given approval to the final version of the manuscript. 


\section{Competing interests}

The authors declare no competing interests.

\section{Additional information}

Supplementary information is available for this paper at https://doi.org/10.1038/s41598-020-59326-3.

Correspondence and requests for materials should be addressed to S.F. or M.R.P.

Reprints and permissions information is available at www.nature.com/reprints.

Publisher's note Springer Nature remains neutral with regard to jurisdictional claims in published maps and institutional affiliations.

(c) (i) Open Access This article is licensed under a Creative Commons Attribution 4.0 International License, which permits use, sharing, adaptation, distribution and reproduction in any medium or format, as long as you give appropriate credit to the original author(s) and the source, provide a link to the Creative Commons license, and indicate if changes were made. The images or other third party material in this article are included in the article's Creative Commons license, unless indicated otherwise in a credit line to the material. If material is not included in the article's Creative Commons license and your intended use is not permitted by statutory regulation or exceeds the permitted use, you will need to obtain permission directly from the copyright holder. To view a copy of this license, visit http://creativecommons.org/licenses/by/4.0/.

(C) The Author(s) 2020 